\title{
Competencies of Graduates of High School for State Exam (K-11) Informatics in Russia
}

\author{
Vladimir M. KIRYUKHIN ${ }^{1}$, Marina S. TSVETKOVA ${ }^{2}$ \\ ${ }^{1}$ Dept. of Informatics and Control Processes, National Research Nuclear University "MEPhI" \\ 31 Kashirskoe Shosse, Moscow 115409, Russian Federation \\ ${ }^{2}$ Academy of Improvement of Professional Skill and Professional Retraining of Educators \\ 8 Golovinskoe Shosse, Moscow 125212, Russian Federation \\ e-mail:vkiryukh@gmail.com,vkiryukhin@mephi.ru,ms-tsv@mail.ru
}

\begin{abstract}
The article presents Codifier elements of the content and competencies of graduates of high school for unified state exam Informatics.
\end{abstract}

Keywords: informatics, computer science, curriculum in informatics at high school (K-11).

\section{Introduction}

The State Exam in Informatics is an exam of choice for high school graduates in Russia (it started in 2008).

Those students who chose a university with an IT profile take the test and its results are taken into account for admission to the university with status of a state exam in mathematics and Russian language.

To prepare for the exam in computer science high school students learn Informatics at the advanced level from 2 to 4 hours per week (a total of 280 hours at 10-11 classes)

The Informatics course includes mathematical foundations of computer science, IT tools and social aspects of information culture.

The exam includes questions on these topics, as well as tasks for the development of algorithms and programming. The examination is conducted in a test form using a computer.

Each version of the examination paper consists of two parts and includes 27 tasks, different forms and levels of complexity. The exam takes a total of 3 hours 55 minutes (FIPI, 2016a).

Part 1 contains a 23 job base, with increased and high levels of difficulty. This part contains a brief reference answer - or in the form of symbol sequence. The test checks all material topics. Part 1: 12 jobs related to baseline for 10 jobs an increased level of complexity, one task - to a high level of complexity. Part 2 contains 4 tasks with detailed answer (FIPI, 2016b). 


\section{Topics of the State Exam in Informatics}

1. Information and Information Processes:

1.1. Information and its Coding:

- Types of information processes.

- The process of transmission of information, the source and the receiver information. Signal encoding and decoding. garbled information.

- Discrete (digital) representation of text, graphics, audio and video information. Units of measurement of the amount of information.

- The speed of information transfer.

1.2. Systems, components, condition and interaction of the components. Information interaction in the system, management, feedback.

1.3. Modeling:

- Description (information model) and the real object of the process, matching the description of the object and the goals. Diagrams, tables, graphs, formulas as descriptions.

- Mathematical models.

- The use of simulation environments (virtual laboratories) for the computer experiment in educational activity.

1.4. Number Systems:

- Positional number system.

- Binary representation of information.

1.5. Logic and algorithms:

- Statements, logical operations, quantifiers, the truth of the statements.

- Chains (finite sequences), trees, lists, graphs, matrices (arrays), pseudo-random sequence.

- Inductive definition objects.

- Computable functions completeness of formalizing the notion of computability, universal computable function.

- Coding.

- Sorting.

1.6. Elements of the theory of algorithms:

- The formalization of the concept of algorithm.

- Computability. The equivalence of algorithmic models.

- Construction of algorithms and practical computations.

1.7. Programming Languages:

- Data Types.

- programming language syntax. programming system.

- The main stages of program development. Breaking tasks into subtasks. 
2. Information Activities:

2.1. Professional information activities. Informational resources.

2.2. Economics of the information sphere.

2.3. Information ethics and law, information security.

3. ICT Tools:

3.1. Architecture of computers and computer networks:

- Software and hardware organization of computers and computer systems. Types of Software.

- Operating Systems. The concept of system administration.

- Safety, hygiene, ergonomics, resource, technological requirements when operating a computer workstation.

3.2. Technologies of creation and processing of textual information:

- The concept of desktop publishing. Creation of computer publications.

- The use of ready-made templates and create your own. Using systems of spelling and grammar. Thesaurus. The use of bilingual translation and electronic dictionaries.

- The use of specialized editing tools of mathematical texts and the graphical representation of mathematical objects.

- The use of OCR systems.

3.3. The creation and processing of graphics and multimedia technology:

- Formats of graphical and sound objects.

- Entering and processing of graphics.

- Entering and processing of audio objects.

3.4. Processing of numerical information:

- The mathematical processing of statistical data.

- Using dynamic (e) tables to perform learning tasks.

- Using tools and solutions statistical calculation and graphic tasks.

3.5. Search technology and information storage:

- Database Management Systems. Database Organization.

- Using tools search engines (querying).

3.6. Telecommunication technologies:

- Special software tools telecommunication technologies.

- Tools create data objects for the Internet.

3.7. Control technology, planning and organization. 


\section{Competences}

\subsection{Know / Uunderstand / Do}

\section{Simulate objects, systems and processes:}

- Calculated in table systems.

- To represent and analyze the information in the tables in the form of charts and graphs.

- Building information models of objects, systems and processes in the form of algorithms.

- Read and debug programs in a programming language.

- Create programs of a description.

- Build models of objects, systems and processes in the form of a truth table for the logical statements.

- Calculate the value of the complex logical statements from the known values of the elementary statements.

\section{Skills in algorithmic problems:}

- Finding a minimum and a maximum of two, three, four numbers of data without the use of arrays and loops.

- Finding all the roots of a given quadratic equation.

- Entry of positive integer positional system with base less than or equal to 10 . The processing and conversion of such a record.

- Finding sums of products of elements of the final numerical sequence (or array).

- The use of cycle for simple search problems (finding the smallest prime divisor of natural numbers, check numbers on simplicity, etc.).

- Filling elements dimensional and two-dimensional arrays on pre-defined rules.

- Operations of the array elements. Linear searching for the item. Insertion and removal of elements in the array. Rearrange the elements of the array in reverse order. The summation of elements in the array. Checking the array elements selected conditions.

- Being the second largest (second maximum or minimum) values in the array for a single view of the array.

- Finding the minimum (maximum) values in the array and the number of elements equal to him for a single view of the array.

- Operation with an array of elements, selected according to a certain condition (for example, finding even a minimum element in the array to find the number and amount of all even elements in the array).

- Sorting an array.

- Merging two ordered arrays into one without sorting.

- Processing of the individual characters of the string. Counting the frequency of occurrence in the character string.

- Working with the substring of this string with the division into words at whitespace. Finding a substring within a given string, the replacement substring with another string. 


\section{Interpret the results of modeling:}

- Use a ready-made model to assess their compliance with the real object and goals of modeling.

- Interpret results obtained during the actual process modeling.

\section{Evaluate the numerical parameters of information objects and processes:}

- Assess the amount of memory required to store information.

- Evaluate the baud rate and data processing.

\subsection{USE Skills in Practice and Everyday Life}

- To carry out the search and selection of information.

- Create and use a data storage structure.

- Work with common automated information systems.

- Prepare and conduct presentations, engage in collective discussion, to fix its course and results of the use of modern software and hardware communications.

- To carry out the statistical processing of the data using a computer.

- To carry out the safety requirements of hygiene, ergonomics and resources when dealing with informatization means.

Performing each task in Part 1 is estimated at 1 point. The task of Part 1 is met if the examinee has answered matching the correct answer. For each task is assigned (in a dichotomous system evaluation), 0 points ("Target"), or 1 point ("task done"). Replies to paragraph 1 are automatically processed after scanning of forms of answers.

The maximum number of initial points is 23 in part 1 .

Completing quests in Part 2 scores from 0 to 4 points. Answers to the tasks in part 2 are tested and evaluated by experts. The maximum number of points is 12 in part 2 .

The maximum score for the test is 35 .

Points for admission to universities are counted on a 100-point scale, which is based on the analysis of the results of the exam performance in Russia this year and recorded in the certificate of high school graduates.

In 2015, the state exam Informatics passed more than 50 thousand high school graduates. On a scale of 100, they showed an average score of 53,6 (Mathematics - 45,4 and Physics - 51,2).

\section{Conclusion}

The winners of the final stage of the All-Russian Olympiad, members of the Russian national teams participating in the International Science Olympiad, at university on a specialty on the profile of the subject of the international competitions, are accepted without entrance examinations. (EGE, 2016). 
The regional stage of the Olympiad was attended by more than 4 thousand students (8-11 classes) from 80 regions of Russia. About 100 participants of these (winners of the final stage) annually receive the right to enter the university without examination.

They are all winners a cash prize of the Russian Government and the Grand - a monthly student scholarship of the President of Russia for the period of study at the university.

The participation of the winners of the Olympic Games in training camp (winter and summer) and a trip to the International Olympiad in Informatics is fully paid by the Russian Government.

\section{References}

FIPI (2016a). The Federal Institute for Education Measurement. (In Russian. Федеральный институт педагогических измерений). Retrieved April 13, 2016, from http://fipi .ru

FIPI (2016b). Open Bank state exam tasks. (In Russian. Открытый банк заданий ЕГЭ). Retrieved April 13, 2016, from http://www.fipi.ru/content/otkrytyy-bank-zadaniy-ege

EGE (2016). Official information portal of the state exam. (In Russian. Официальный Информационный Портал Единого Государственного Экзамена). Retrieved April 13, 2016, from

http: //www.ege.edu.ru
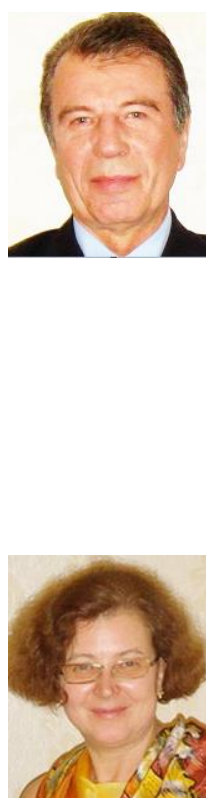

V.M. Kiryukhin is professor of the Russian Academy of Natural Sciences. He is the chairman of the federal methodical commission on informatics which is responsible in Russia for carrying out the national Olympiads in informatics. He is the author of many papers and books in Russia on development of Olympiad movements in informatics and preparations for the Olympiads in informatics. He is the exclusive representative who took part at all IOI from 1989 as a member of the IOI International Committee (1989-1992, 1999-2002, 20132016) and as the Russian team leader (1989, 1993-1998, 2003-2012). He received the IOI Distinguished Service Award at IOI 2003, the IOI Distinguished Service Award at IOI 2008 as one of the founders of the IOI making his long term distinguished service to the IOI from 1989 to 2008 and the medal "20 Years since the First International Olympiad in Informatics" at the IOI 2009.

M.S. Tsvetkova, professor of the Russian Academy of Natural Sciences, $\mathrm{PhD}$ in pedagogic science, prize-winner of competition "The Teacher of Year of Moscow" (1998), main expert of state projects of school education informatization in the Ministry of Education of the Russian Federation (2001-2005), the expert of the World bank project "Informatization of Education System". Since 2002 she is a member of the Central methodical commission of the Russian Olympiad in informatics, the pedagogic coach of the Russian team on the IOI. She is the author of many papers and books in Russia on the informatization of education and methods of development of talented students. Since 2013 she is the Russian team leader. 